\title{
On the role of dynamical quark mass generation in chiral symmetry breaking in QCD
}

\author{
H. Sazdjian \\ Institut de Physique Nucléaire, CNRS/IN2P3, \\ Université Paris-Sud, F-91405 Orsay, France
}

\begin{abstract}
The phenomenon of dynamical quark mass generation is studied in QCD within the framework of a gauge invariant formalism. An exact relationship is established between the equation satisfied by the scalar part of the two-point gauge invariant quark Green's function and the quark-antiquark bound state equation in the chiral limit. A possible nontrivial solution of the former yields a massless pseudoscalar solution of the bound state equation with vanishing total momentum. The result is also corroborated by the corresponding Ward-Takahashi identity. The problem is explicitly solved in two-dimensional QCD in the large- $N_{c}$ limit.
\end{abstract}

Keywords:

QCD, quark, dynamical mass generation, chiral symmetry breaking, Wilson loop, gauge invariant Green's function

\section{Introduction}

It has been known for a long time, since the advent of the Nambu-Jona-Lasinio model [1], that a mechanism of chiral symmetry breaking in theories with fermions as the elementary matter fields is provided by the dynamical mass generation phenomenon. This mechanism was also analyzed in the framework of QED by Baker, Johnson and Lee [2], but the presence of the axial-vector current anomaly in Abelian sectors of the chiral group did not allow drawing a similar conclusion.

The problem was later studied in the framework of QCD, with the aid of models applied to the DysonSchwinger equations [3, 4, 5, 6] in the Coulomb gauge, where the gluon propagator in the rainbow-ladder approximation is considered with its instantaneous expression, with an appropriate confining behavior [7, 8, 9, 10]. The dynamical mass generation phenomenon was confirmed and phenomenological applications to the meson spectrum were considered. An extension of the approximation beyond the rainbow-ladder one preserved the stability of the phenomenon [11]. On the other hand, the use of the Ward-Takahashi identity for the axial-vector current [12] establishes a general relationship, independent of approximation schemes of the

Email address: sazdjian@ipno.in2p3.fr (H. Sazdjian)
Bethe-Salpeter kernel, between the dynamically generated quark mass and the pseudoscalar Goldstone boson wave function [13].

In spite of these positive results, the notion of dynamical quark mass remains ambiguous. Contrary to the case of nonconfining theories, the quark mass is not an observable quantity. Furthermore, the quark propagator not being a gauge invariant object, it is difficult to give a physical interpretation to its singularities. In many examples, an infrared cutoff is needed to make it finite.

The aim of this talk is to present results obtained within a gauge invariant formalism, developed in recent years [14, 15], where an exact relationship is established between the dynamical quark mass term and the pseudoscalar Goldstone boson wave function in the total zero momentum limit. The explicit resolution of the problem in analytic form in two-dimensional QCD in the large- $N_{c}$ limit illustrates the advantage of the gauge invariant approach in providing a physical insight into the interpretation of the dynamical mass generation phenomenon.

\section{Gauge invariant quark Green's functions}

To define gauge invariant quark Green's functions, one needs to introduce gluon field path-ordered phase factors (Wilson lines) [16, 17], which are the operators 
of parallel transport of fields from one point to the other. The paths followed by the phase factors might be arbitrarily chosen, however, for the quark Green's functions polygonal lines seem to correspond to the optimal choice. First, they can be decomposed along a succession of straight line segments. The latter are Lorentz invariant in form and this in turn allows the classification of the polygonal lines according to the number of segments they contain. Second, polygonal lines form a complete set of lines for the description of the present problem, in the sense that no other types of line are needed to complete the description.

The classification adopted for the polygonal lines is applied to the two-point gauge invariant quark Green's functions (2PGIQGF). We designate by $U(y, x)$ the path-ordered phase factor along the oriented straight line segment going from $x$ to $y$. The quark fields, with mass parameter $m$, are assumed to belong to the defining fundamental $\left(N_{c}\right.$-dimensional) representation of the color gauge group $S U\left(N_{c}\right)$. The 2PGIQGF with a polygonal line composed of the succession of $n$ segments with $(n-1)$ junction points is designated by $S_{(n)}$ and defined as

$$
\begin{gathered}
S_{(n)}\left(x, x^{\prime} ; t_{n-1}, \ldots, t_{1}\right)=-\frac{1}{N_{c}}\left\langle\bar{\psi}\left(x^{\prime}\right) U\left(x^{\prime}, t_{n-1}\right)\right. \\
\left.\times U\left(t_{n-1}, t_{n-2}\right) \ldots U\left(t_{1}, x\right) \psi(x)\right\rangle .
\end{gathered}
$$

(The vacuum expectation value is defined in the path integral formalism; spinor indices are omitted and the color indices are implicitly summed.) The simplest such function is $S_{(1)}$, having a phase factor along one straight line segment:

$$
S_{(1)}\left(x, x^{\prime}\right)=-\frac{1}{N_{c}}\left\langle\bar{\psi}\left(x^{\prime}\right) U\left(x^{\prime}, x\right) \psi(x)\right\rangle .
$$

Use of the equations of motion of Green's functions and integrations yield functional relations between the Green's functions of different classes of polygonal line. It turns out that $S_{(1)}$ is the only dynamically independent 2PGIQGF. All other 2PGIQGFs $S_{(n)}$, with $n>1$, are calculable from $S_{(1)}$ through the functional relations. These involve essentially Wilson loops [18] along polygonal contours and functional derivatives acting on their sides.

The equation satisfied by $S_{(1)}$ can be brought to the following form:

$$
\begin{aligned}
\left(i \gamma . \partial_{(x)}-\right. & m) S_{(1)}\left(x, x^{\prime}\right)=i \delta^{4}\left(x-x^{\prime}\right)+i \gamma^{\mu}\left\{K_{1 \mu}\left(x^{\prime}, x\right)\right. \\
& \times S_{(1)}\left(x, x^{\prime}\right)+K_{2 \mu}\left(x^{\prime}, x, y_{1}\right) S_{(2)}\left(y_{1}, x^{\prime} ; x\right) \\
& +\sum_{n=3}^{\infty} K_{n \mu}\left(x^{\prime}, x, y_{1}, \ldots, y_{n-1}\right)
\end{aligned}
$$

$$
\left.\times S_{(n)}\left(y_{n-1}, x^{\prime} ; x, y_{1}, \ldots, y_{n-2}\right)\right\},
$$

where the kernels $K_{n}(n=1,2, \ldots)$ contain Wilson loop averages along polygonal contours, which are at most $(n+1)$-sided, and $(n-1) 2$ PGIQGFs $S_{(1)}$ and their derivative. The total number of derivatives contained in $K_{n}$ is $n$, each derivative acting on a different segment. Since the high-index $S_{(n)}$ s can be expressed in terms of $S_{(1)}$, Eq. (3) is an integrodifferential equation in $S_{(1)}$, which is the primary unknown quantity to be solved. (Integration symbols on the intermediate variables have been omitted in the right-hand side.) Equation (3) is the ana$\log$ of the self-energy Dyson-Schwinger equation in the present formalism.

\section{Bound state wave functions}

The previous definitions and approach can also be applied to four-point Green's functions. We introduce two quark fields (in flavor space) $\psi_{1}$ and $\psi_{2}$, with masses $m_{1}$ and $m_{2}$, respectively, and consider gauge invariant fourpoint Green's functions made of two quark and two antiquark fields and appropriate phase factors along polygonal lines. Considering a mesonic bound state, with total momentum $P$, one defines corresponding gauge invariant wave functions with a similar classification as for the 2PGIQGFs $(n=1,2, \ldots)$ :

$$
\begin{aligned}
\Phi_{(n)}(P ; & \left.x_{1}, x_{2} ; t_{n-1}, t_{n-2}, \ldots, t_{1}\right)= \\
& -\frac{1}{\sqrt{N_{c}}}<0 \mid T\left(\bar{\psi}_{2}\left(x_{2}\right) U\left(x_{2}, t_{n-1}\right)\right. \\
& \left.\times U\left(t_{n-1}, t_{n-2}\right) \ldots U\left(t_{1}, x_{1}\right) \psi_{1}\left(x_{1}\right)\right) \mid P>.
\end{aligned}
$$

In the simplest case $n=1$, one has

$$
\begin{aligned}
& \Phi_{(1)}\left(P ; x_{1}, x_{2}\right)= \\
& -\frac{1}{\sqrt{N_{c}}}<0\left|T\left(\bar{\psi}_{2}\left(x_{2}\right) U\left(x_{2}, x_{1}\right) \psi_{1}\left(x_{1}\right)\right)\right| P>.
\end{aligned}
$$

The above wave functions, for all $n \mathrm{~s}$, describe the same bound state, but differ in their expressions due to their differences in their contents with respect to the phase factor lines.

As in the case of the 2PGIQGFs, functional relations exist between the above wave functions: $\Phi_{(n)}$ s, with $n>$ 1 , are calculable in terms of $\Phi_{(1)}$.

The wave function $\Phi_{(1)}$ satisfies two Dirac-type integrodifferential equations, which can be written in the following compact forms:

$$
\left(i \gamma . \partial_{1}-m_{1}\right) \Phi_{(1)}\left(P ; x_{1}, x_{2}\right)=+i \gamma^{\mu}\left\{\sum_{n=1}^{\infty} K_{1, n \mu} * \Phi_{(n)}\right.
$$




$$
\begin{gathered}
\left.+\sum_{n=1}^{\infty}\left(N_{1, n \mu} * \Phi_{(1)}\right) * S_{2(n)}\right\} \\
\Phi_{(1)}\left(P ; x_{1}, x_{2}\right)\left(-i \gamma . \overleftarrow{\leftarrow}_{2}-m_{2}\right)=-i\left\{\sum_{n=1}^{\infty} \Phi_{(n)} * K_{2, n v}\right. \\
\left.+\sum_{n=1}^{\infty} S_{1(n)} *\left(\Phi_{(1)} * N_{2, n v}\right)\right\} \gamma^{v} .
\end{gathered}
$$

The kernels $K_{n}$ are similar to those met in Eq. (3); they contain Wilson loop averages along polygonal contours with $(n+1)$ sides; the additional indices 1 or 2 reflect the differences that arise from the presence there of the $(n-1) 2$ PGIQGFs $S_{(1)}$ of quarks 1 and/or 2 . The kernels $N_{n}$, with indices 1 or 2 , are obtained from the $K_{n}$ s by functional derivation with respect to one of the 2PGIQGFs. The star operations are compact notaions for the various integration operations on the intermediate variables. Since the wave functions $\Phi_{(n)}$ are calculable in terms of $\Phi_{(1)}$, the above equations are ultimately wave equations for $\Phi_{(1)}$.

\section{Chiral symmetry breaking}

To analyze the chiral symmetry breaking possibility, we consider the 2PGIQGF $S_{(1)}\left(x_{1}, x_{2}\right)$. Since the single straight line segment that is present in the path-ordered phase factor depends only on the end points $x_{1}$ and $x_{2}$, we can decompose $S_{(1)}$ along two Poincaré invariant parts, made of a vector and of a scalar:

$$
S_{(1)}(x)=i \gamma . \partial F_{1}\left(x^{2}\right)+F_{0}\left(x^{2}\right) .
$$

$\left(x=x_{1}-x_{2}\right.$.) In perturbation theory, the scalar component $F_{0}$ is proportional to the quark mass $m$. When the latter vanishes, $F_{0}$ also vanishes. Therefore, if the solution of the equation of $S_{(1)}$ yields a scalar part that does not vanish in the limit of a massless quark, then one is in the presence of a nonperturbative solution, which may be described as corresponding to the phenomenon of dynamical mass generation. This mass term might have a complicated structure, neither being a constant nor producing a simple pole in the 2 PGIQGF.

The equation satisfied by $F_{0}$ can be extracted from that of $S_{(1)}$ [Eq. (3)] by considering the anticommutator of $\gamma_{5}$ with $S_{(1)}$. One obtains in the massless quark limit

$$
\begin{gathered}
i \gamma . \partial_{1}\left[\gamma_{5}, S_{(1)}\left(x_{1}, x_{2}\right)\right]_{+}=i \gamma^{\mu}\left\{\sum_{n=1}^{\infty} K_{n \mu} *\left[\gamma_{5}, S_{(n)}\right]_{+}\right. \\
\left.+\sum_{n=1}^{\infty}\left(N_{n \mu} *\left[\gamma_{5}, S_{(1)}\right]_{+}\right) * S_{(n)}\right\} .
\end{gathered}
$$

This equation is to be compared with that of the wave function $\Phi_{(1)}$ in the massless limit:

$$
\begin{gathered}
i \gamma . \partial_{1} \Phi_{(1)}\left(P ; x_{1}, x_{2}\right)=i \gamma^{\mu}\left\{\sum_{n=1}^{\infty} K_{n \mu} * \Phi_{(n)}\right. \\
\left.+\sum_{n=1}^{\infty}\left(N_{n \mu} * \Phi_{(1)}\right) * S_{(n)}\right\} .
\end{gathered}
$$

(The quark indices 1 and 2 have been removed, since the quarks are massless.) Equations similar to (9) and (10) with operators acting from the right on the variable $x_{2}$ could also be written down.

We observe that the equation of $\left[\gamma_{5}, S_{(1)}\right]_{+}$is the same as the equation of $\Phi_{1)}$, provided the general correspondences $\left[\gamma_{5}, S_{(n)}\right]_{+} \longrightarrow \Phi_{(n)}$ are also made. Actually, these correspondences are independently justified by comparing the equations each of them satisfy. They represent the general consistency conditions for the similarity of the set of all equations of the above types.

Therefore, if the equation of $S_{(1)}$ has, in the massless quark limit, a nonvanishing solution for $\left[\gamma_{5}, S_{(1)}\right]_{+}$, then this is also a solution of the bound state equation of $\Phi_{(1)}$ for a pseudoscalar state with zero total momentum $P$, since $S_{(1)}$ does not depend on $P$. This is possible only if $P^{2}=0$; this means that the pseudoscalar state is massless and represents a Goldstone boson for chiral symmetry breaking.

A few comments are in order here. First, the above results are exact statements of QCD, although for unrenormalized quantities, since no approximations were made throughout the calculations; all expressions of the kernels are explicitly known in terms of Wilson loop averages and functional derivatives. Second, one may adopt, at the practical level of resolution of the equations, an approximation scheme based mainly on the truncation of the series of kernels according to the number of sides of the polygonal contours of the Wilson loops. There are indications that the series expansions in the right-hand sides of the integrodifferential equations are perturbative with respect to the inverse of the number of sides of polygonal contours. Actually, the first kernels $K_{1}$ and $N_{1}$ vanish for symmetry reasons and therefore the leading kernels are $K_{2}$ and $N_{2}$, corresponding to triangular Wilson loops. In any event, to maintain the consistency between the various equations, one should adopt the same type of approximation in all equations. Third, the conclusions that are drawn are gauge invariant, since all quantities under consideration are individually gauge invariant and this property is not altered by the approximation scheme of the truncation type. This is one of the advantages of the gauge invariant formalism. 


\section{Ward-Takahashi identities}

Ward-Takahashi identities (WTI) express in compact form relations among Green's functions that emerge from symmetry properties of a theory. Here we sketch the WTI adapted to the gauge invariant formalism that we are using and corresponding to chiral symmetry.

Considering the case of two different quarks, 1 and 2 , we define the axial-vector current $j_{\mu 5}^{12}$ and the pseudoscalar density $v^{12}$ as

$$
j_{\mu 5}^{12}(y)=\bar{\psi}_{1}(y) \gamma_{\mu} \gamma_{5} \psi_{2}(y), \quad v^{12}(y)=i \bar{\psi}_{1}(y) \gamma_{5} \psi_{2}(y),
$$

and introduce the full axial-vector and pseudoscalar vertex functions

$$
\begin{aligned}
& J_{\mu 5, \alpha \beta}^{12}\left(x_{1}, x_{2} ; y\right)=-\frac{1}{N_{c}}\left\langle\bar{\psi}_{2 \beta}\left(x_{2}\right) U\left(x_{2}, x_{1}\right) \psi_{1 \alpha}\left(x_{1}\right) j_{\mu 5}^{12}(y)\right\rangle, \\
& P_{\alpha \beta}^{12}\left(x_{1}, x_{2} ; y\right)=-\frac{1}{N_{c}}\left\langle\bar{\psi}_{2 \beta}\left(x_{2}\right) U\left(x_{2}, x_{1}\right) \psi_{1 \alpha}\left(x_{1}\right) v^{12}(y)\right\rangle .
\end{aligned}
$$

( $\alpha, \beta$ are the quark spinor indices.) The WTI takes the form

$$
\begin{aligned}
& \partial_{y}^{\mu} J_{\mu 5}^{12}\left(x_{1}, x_{2} ; y\right)=\left(m_{1}+m_{2}\right) P^{12}\left(x_{1}, x_{2} ; y\right) \\
& -\gamma_{5} S_{2(1)}\left(x_{1}, x_{2}\right) \delta^{4}\left(y-x_{1}\right)-S_{1(1)}\left(x_{1}, x_{2}\right) \gamma_{5} \delta^{4}\left(y-x_{2}\right)
\end{aligned}
$$

The analysis that follows is then standard. One passes to momentum space, where only two momenta are independent; the total momentum $P$ enters in the singularities of the vertex functions coming from the mesonic sector. One first considers the chiral limit $m_{1}=m_{2}=0$, which removes the first term of the right-hand side of Eq. (14) and transforms, in the limit $P \rightarrow 0$, the contributions of the 2PGIQGFs into a term proportional to the anticommutator of $S_{(1)}$ with $\gamma_{5}$. If the latter contribution does not vanish in these limits, then it should be compensated by the left-hand side. This is possible only if the axial-vector vertex function possesses a pseudoscalar pole at $P^{2}=0$. The residue of this pole is proportional to the wave function of the state and to the weak decay constant $F_{P}$ of the Goldstone boson. One thus finds the proportionality relation between the Goldstone boson wave function at $P=0$ and the scalar part of the 2PGIQGF.

In the next step, one considers the case where the quark masses are different from zero and takes the limit of $P^{2}$ to the physical mass of the would-be Goldstone boson. In this limit, only the two vertex functions of Eq. (14) survive. One then considers an expansion in the pseudoscalar vertex residue in terms of the quark masses, keeping only the leading contribution. Remembering that the Goldstone boson wave function is proportional to the scalar part of the 2PGIQGF, one ends up with the Gell-Mann-Oakes-Renner relation [19]

$$
m_{P}^{2} F_{P}^{2}=-\left(m_{1}+m_{2}\right)<\bar{q} q>_{0},
$$

where $<\bar{q} q>_{0}$ is the quark condensate in the chiral limit per flavor unit.

\section{Two-dimensional QCD}

Two-dimensional QCD in the large- $N_{c}$ limit is a simplified laboratory for real QCD [20, 21]. It possesses the main characteristics of confinement and allows the study of the infrared behavior of the theory in a more explicit way. On the other hand, Wilson loop averages can be explicitly calculated: they satisfy the area law, due to the fact that the coupling constant of the theory is dimensionful from the start [22, 23, 24].

Equation (3) has also its counterpart in two dimensions. It turns out that the kernels $K_{n}$ for $n>2$ vanish due to the particular structure of the Wilson loop averages. ( $K_{1}$ vanishes in general for symmetry reasons.) One remains solely with the kernel $K_{2}$. Equation (3) then reduces to a nonlinear nonlocal equation in $S_{(1)}$. The equation can be solved explicitly and analytically by analyzing the singularity structure of the 2PGIQGF [25].

The solution is infrared finite with singularities in momentum space lying on the positive real axis of $p^{2}$ (timelike region). They are represented by an infinite number of branch points, characterized by positive masses $M_{n}(n=1,2, \ldots)$ with threshold singularities equal to $-3 / 2$ in $M_{n}^{2}$. The expression of $S_{(1)}$ is, in momentum space,

$$
S_{(1)}(p)=-i \frac{\pi}{2 \sigma} \sum_{n=1}^{\infty} b_{n} \frac{\left(\gamma \cdot p+(-1)^{n+1} M_{n}\right)}{\left(M_{n}^{2}-p^{2}\right)^{3 / 2}},
$$

where $\sigma$ is the string tension. The masses $M_{n}$ are greater than the free quark mass $m$ and ordered according to increasing values. For massless quarks they remain positive. The masses $M_{n}$ and the coefficients $b_{n}$, the latter being also positive, satisfy, for general $m$, an infinite set of algebraic equations that are solved numerically. Their asymptotic values, for large values of $n$ such that $n \gg m^{2} /(\pi \sigma)$, are

$$
M_{n}^{2} \simeq \pi n \sigma, \quad b_{n} \simeq \frac{\sigma^{2}}{M_{n}+(-1)^{n} m} .
$$


The masses $M_{n}$ can be considered as dynamically generated quark masses, since they do not exist in the QCD Lagrangian. The gauge invariant treatment of the problem has displayed two main features: First, the dynamically generated masses are infinite in number. Second, they do not produce simple poles in the quark Green's functions, but stronger singularities; this fact might prevent quarks from being observed as asymptotic free states.

The appearance of dynamically generated masses is also accompanied by a nonvanishing value of the quark condensate in the chiral limit. Since the quark condensate is a local gauge invariant quantity, it can also be calculated in various gauges. Calculations done in the light cone gauge [26, 27], in the axial gauge [28] and in the present approach [25], give numerically the same result.

\section{Conclusion}

Use of a gauge invariant formalism has confirmed the general relationship that exists in QCD between the wave function of the pseudoscalar Goldstone boson of chiral symmetry breaking and the scalar part of the twopoint gauge invariant quark Green's function, in case the latter is nonvanishing in the chiral limit. The relationship also persists under truncation schemes of the exact interaction kernels.

Two-dimensional QCD in the large- $N_{c}$ limit, provides an explicit illustration of the above phenomenon. The dynamical quark mass generation produces an infinite number of branch point singularities, stronger than simple poles, which are infrared finite and describe the mechanism of chiral symmetry breaking in that theory.

\section{References}

[1] Y. Nambu, G. Jona-Lasinio, Dynamical model of elementary particles based on an analogy with superconductivity. I, Phys. Rev. 122 (1961) 345.

[2] M. Baker, K. Johnson, B. W. Lee, Broken symmetries and zeromass bosons, Phys. Rev. 133 (1964) B209.

[3] F. J. Dyson, The S matrix in quantum electrodynamics, Phys. Rev. 75 (1949) 1736.

[4] J. S. Schwinger, On the Green's functions of quantized fields. 1., Proc. Nat. Acad. Sci. 37 (1951) 452.

[5] R. Alkofer, L. von Smekal, The infrared behavior of QCD Green's functions, Phys. Rept. 353 (2001) 281.

[6] C. S. Fischer, Infrared properties of QCD from DysonSchwinger equations, J. Phys. G 32 (2006) R253.

[7] J. Finger, J. E. Mandula, J. Weyers, The pion in QCD, Phys. Lett. B96 (1980) 367

[8] A. Le Yaouanc, L. Oliver, O. Pène, J.-C. Raynal, Spontaneous breaking of chiral symmetry for confining potentials, Phys. Rev. D 29 (1984) 1233.
[9] S. L. Adler, A. C. Davis, Chiral symmetry breaking in Coulomb gauge QCD, Nucl. Phys. B 244 (1984) 469.

[10] R. Alkofer, P. A. Amundsen, Chiral symmetry breaking in an instantaneous approximation to Coulomb gauge QCD, Nucl. Phys. B 306 (1988) 305.

[11] A. Bender, C. D. Roberts, L. von Smekal, Goldstone theorem and diquark confinement beyond rainbow ladder approximation, Phys. Lett. B 380 (1996) 7.

[12] G. Preparata, W. I. Weisberger, Ultraviolet divergences in radiative corrections to weak decays, Phys. Rev. 175 (1968) 1965.

[13] P. Maris, C. D. Roberts, P. C. Tandy, Pion mass and decay constant, Phys. Lett. B 420 (1998) 267.

[14] H. Sazdjian, Integral equation for gauge invariant quark twopoint Green's function in QCD, Phys. Rev. D 77 (2008) 045028.

[15] H. Sazdjian, Gauge invariant bound state equations for quarkantiquark systems in QCD, Phys. Rev. D 88 (2013) 025034.

[16] S. Mandelstam, Feynman rules for electromagnetic and YangMills fields from the gauge independent field theoretic formalism, Phys. Rev. 175 (1968) 1580.

[17] Y. Nambu, QCD and the string model, Phys. Lett. B80 (1979) 372.

[18] K. G. Wilson, Confinement of quarks, Phys. Rev. D10 (1974) 2445.

[19] M. Gell-Mann, R. J. Oakes, B. Renner, Behavior of current divergences under $S U(3) \times S U(3)$, Phys. Rev. 175 (1968) 2195 .

[20] G. 't Hooft, A planar diagram theory for strong interactions, Nucl. Phys. B 72 (1974) 461.

[21] G. 't Hooft, A two-dimensional model for mesons, Nucl. Phys. B 75 (1974) 461.

[22] V. A. Kazakov, I. K. Kostov, Nonlinear strings in twodimensional $U(\infty)$ gauge theory, Nucl. Phys. B 176 (1980) 199.

[23] V. A. Kazakov, Wilson loop average for an arbitrary contour in two-dimensional $U(N)$ gauge theory, Nucl. Phys. B 179 (1981) 283.

[24] N. E. Bralic, Exact computation of loop averages in twodimensional Yang-Mills theory, Phys. Rev. D 22 (1980) 3090.

[25] H. Sazdjian, Spectral properties of the gauge invariant quark Green's function in two-dimensional QCD, Phys. Rev. D 81 (2010) 114008

[26] A. R. Zhitnitsky, On chiral symmetry breaking in QCD in twodimensions $\left(N_{c} \rightarrow \infty\right)$, Phys. Lett. B165 (1985) 405 .

[27] M. Burkardt, Trivial vacua, high orders in perturbation theory and nontrivial condensates, Phys. Rev. D 53 (1996) 933.

[28] M. Li, Large $N$ two-dimensional QCD and chiral symmetry, Phys. Rev. D 34 (1986) 3888 